\title{
Identitätssuche bei Flüchtlingskindern am Beispiel von Peter Härtlings Roman «Djadi, Flüchtlingsjunge»
}

\section{Ehab Mokhtar Khalaf Abdel-Hafiz}

German department, Al-Alsun faculty, Al-Minia University, Minia, Egypt

ABSTRACT

ARTICLE INFO

Received

Accepted

Keywords

Identity, Refugees, War, Ethnicity, Homeland
Based on Peter Härtling's novel "Djadi, Refugee Boy", this research examines ego and social identity of the fictional character "Djadi". Children's ego identity is often shaken by traumatic experiences. Härtling says from his own experience that refugees in the host country must first be received as people in need. Only when a new basis of trust has been built in the new society, can the process of integration begin. The definition and development of identity are the ground of this work. A healthy self-identity plays an important role in the integration of refugee children. In this case, it helps the children to know who they actually are and what is expected of them in their new society. Social identity is of particular importance in this field. The host society should be patient and conscious with such children. The question of one's own identity is above all 
when one is confronted with other cultures and different points of view. According to Erikson, it is very important, that children acquire a positive sense of values in order to find a place in the society around them. So, they can become good members, no fanatics. The traumatized children because of warrelated traumatic events need additional support to integrate into the new country successfully. Peter Härtling tells in his novel the story of an unaccompanied minor, so that the readers can better understand the problem of unaccompanied immigrant minors and develop empathy towards them.

\section{Biographie des Autors}

Peter Härtling, ein bekannter und beliebter Schriftsteller Deutschlands hat fast 30 Romane und Erzählungen geschrieben, dazu Dramen, Gedichte, Essays und Kinderbücher und wurde mit vielen Auszeichnungen und Ehrungen bedacht. Er kam 1933 in Chemnitz zur Welt. Sein Vater war Rechtsanwalt. Im Alter von sechs Jahren zog seine Familie wegen den Nationalsozialisten nach Olmütz in Mähren. Gegen Ende des zweiten Weltkrieges mussten sie vor der Roten Armee nach Zwettl in Niederösterreich fliehen. Dort geriet Peter Härtlings Vater 1945 in die russische Gefangenschaft und starb. Seine Mutter wurde im Beisein ihres Sohnes von russischen Soldaten vergewaltigt. Kurz darauf übersiedelte Peter Härtling, mit seiner Mutter, Großmutter, Tante und seiner kleinen Schwester nach Nürtingen bei Württemberg. Er besuchte dort das Max-Planck-Gymnasium. 1946 nahm sich seine Mutter das Leben. Härtling war mit 13 Jahren Vollwaise und wurde von Verwandten aufgenommen. Er verlässt das Gymnasium noch 
vor Abschluss des Abiturs und beginnt mit journalistischen Tätigkeiten. Schon in jungen Jahren interessierte er sich für Literatur und war ein Vielleser. 1959 heiratete er die Psychologin Mechthild Maier. Das Paar hat vier gemeinsame Kinder. 1967 wird Peter Härtling Cheflektor und übernimmt danach die Geschäftsleitung des S. Fischer Verlags in Frankfurt am Main. Seit 1974 arbeitet er als freier Schriftsteller. Seine Texte sind geprägt von autobiographischen Elementen. Flucht und Vertreibung sind Schlüsselerfahrungen in Peter Härtlings eigenem Leben und spielen auch in seinen Büchern immer wieder eine Rolle. Durch das Schreiben verarbeitet er seine schwierige Kindheit, seine problematische Beziehung $\mathrm{zu}$ seinem Vater und die Fluchtereignisse. Peter Härtling starb im Alter von 83 Jahren im Juli 2017 in Rüsselheim, Deutschland. ${ }^{1}$

Als Peter Härtling 2015 das Bild eines kleinen syrischen Flüchtlingsjungen in den Medien sah, der tot an einem Strand im Süden der Türkei gefunden wurde, bewegte ihn dies dazu, das Buch «Djadi, Flüchtlingsjunge» zu schreiben. Die Flüchtlingskrise wühlte ihn sehr auf, da viele Erinnerungen an die eigene Flucht in ihm wieder wach wurden. Mit seinem Roman wollte er Kindern und Erwachsenen erklären, wie sie mit traumatisierten und verschlossenen Flüchtlingskindern umgehen können. In einem Interview sagte er:

«Es hätte mir als Kind geholfen, wenn man mich nicht als ein Niemand, der integriert werden muss, behandelt hätte -sondern als Mensch.»

Für die Recherche hat Härtling viele Berichte von und über Flüchtlingskinder aus Syrien und Afghanistan gelesen. Zudem beriet ihn ein Sohn, der Kinder- und Jugendpsychiater ist. Die

\footnotetext{
${ }^{1}$ Hans H. Ewers, 2005, Mitleid für das eigene »Kind in mir«, https://www.forschungfrankfurt.uni-frankfurt.de/36050401/forschung-frankfurt-ausgabe-2-2005-mitleidfur-das-eigene-kind-in-mir.pdf und http://www.haertling.de/

${ }^{2}$ Franziska Annabelle Lindner, 2016,

https://www.beltz.de/fileadmin/user_upload/PI_Haertling-Djadi.pdf
} 
eigenen Erfahrungen der Kindheit, so wie die Bücher von Silke Beckert, «Unbegleitete minderjährige Flüchtlinge in der Kinderund Jugendhilfe» und Brigitte Haargasser, «UmF. Sequenzielle Traumatisierung und die Aufgaben der Jungenhilfe» dienten als Grundlagen für seinen Roman. ${ }^{3}$ Ein Thema, das Peter Härtling in diesem Buch aufnimmt, beschreibt die Tatsache, dass bei einem Kind die bisher entwickelte Ich-Identität, durch die traumatischen Ereignisse eines Krieges, stark leidet oder sogar zerstört werden kann. In diesem Zusammenhang stellt sich die Frage, was genau bedeutet «Identität»?

\section{Der theoretische Teil}

Die Identität beschreibt die Antwort auf folgende Fragen: «Wie bin ich geworden, was ich bin?», «Wer bin ich, wer will ich sein?», «Was tue ich?» und «Wie sehen mich die anderen?» 4 definieren:

Den Begriff der Identität kann man laut H. Abels wie folgt

«Identität ist das Bewusstsein, ein unverwechselbares Individuum mit einer eigenen Lebensgeschichte zu sein, in seinem Handeln eine gewisse Konsequenz zu zeigen und in der Auseinandersetzung mit anderen eine Balance zwischen individuellen Ansprüchen und sozialen Erwartungen gefunden zu haben.» 5

Oft setzten sich Menschen erst bewusst mit der eigenen Identität auseinander, wenn sie mit der Frage konfrontiert werden: «Wer bist du?» oder «Wer sind Sie?» Normalerweise wird die Frage primär mit Fakten der personalen Identität beantwortet. Man nennt seinen Namen, das Alter, die Nationalität, den Beruf etc. Es sind die Daten, die auch in den offiziellen Papieren einer Person

\footnotetext{
${ }^{3}$ Kristina Kroll, 2017, «Djadi, Flüchtlingsjunge» im Unterricht, Beltz \& Gelberg Verlag, Weinheim, S. 19

${ }^{4}$ Heinz Abels, 2006, Identität, VS Verlag für Sozialwissenschaften, Wiesbaden, S. 245

5 ebd, S. 254
} 
vermerkt sind, anhand derer jemand identifiziert werden kann. Danach wird oft die Gegenfrage gestellt: «Was möchtest du sonst noch wissen?» oder «Was möchten Sie sonst noch wissen?». 6

Die Identität setzt sich aus drei Komponenten zusammen: der personalen Identität (I), der sozialen Identität (me) und der IchIdentität (self). Alle drei Komponenten beeinflussen sich gegenseitig. Die personale Identität (I) umfasst Persönlichkeitsmerkmale wie die Charakteristika des Körpers, die Fähigkeiten, die Interessen und die Biographie. Auch spontanes, unreflektiertes und impulsives Handeln zählt dazu. All diese Gegebenheiten machen die Einzigartigkeit eines Menschen aus. Die personale Identität verleiht dem Individuum die Individualität, ist sowohl genetisch beeinflusst als auch sozial überformt. ${ }^{7}$ Die personale Identität der fiktiven Figur «Djadi» wird im Roman von Peter Härtling wie folgt beschrieben: er ist zu klein für sein Alter von 11 Jahren, zu dünn, krummbeinig, hat ein angestrengtes Gesicht mit großen schwarzen Augen und kommt aus Syrien. (S.5/18) $\mathrm{Zu}$ seinen unreflektierten Angsthandlungen gehört, sich bei Unsicherheit, wie eine flüchtende Eidechse, blitzartig unter dem Sofa zu verstecken. (S.8/12)

Die soziale Identität (me) setzt sich auseinander mit den Fragen: «Welche soziale Rolle übernehme ich? Welcher Gruppe gehöre ich an? Was wird von mir erwartet? Was denken die anderen Menschen von mir?». Das Individuum entwickelt sich immer in Interaktion mit anderen Menschen, deshalb spielt das soziale Milieu, in dem es aufwächst, eine wesentliche Rolle. Es besteht eine dauerhafte Wechselwirkung zwischen der Gesellschaft und den Anlagen, die jeder Mensch von Geburt an mitbringt. Durch Kommunikation und Interaktionen mit den unterschiedlichsten

\footnotetext{
${ }^{6}$ Jörg Zirfas, 2010, Schlüsselwerke der Identitätsforschung, VS Verlag für Sozialwissenschaften, Wiesbaden, S. $5 \mathrm{ff}$

${ }^{7}$ Bernadette Müller, 2011, Empirische Identitätsforschung, VS Verlag für Sozialwissenschaften, Wiesbaden, S. 37/74
} 
Menschen und Gruppen erlangt jede Person ein Bild von sich selbst und seinem Umfeld. Positive Feedbacks zur eigenen Person beflügeln das Selbstwertgefühl, machen stolz und stärken die Persönlichkeit. Es liegt in der Natur des Menschen, dass er respektiert, anerkannt und geliebt werden möchte. Negative Rückmeldungen, Erniedrigungen und Demütigungen lösen Schamgefühle aus. Das eigene Wohlbefinden und das Selbstwertgefühl sinken. Dies kann soweit führen, dass sich ein Mensch zur Selbsttötung entschliesst. ${ }^{8}$

Sozialstrukturelle Merkmale, wie die sozialen Rollen einer Person (z.B. Lehrerin, Mutter, Patientin) beeinflussen die soziale Identität. Eine Mutter zum Beispiel identifiziert sich meist über ihre Familie, eine Politikerin hingegen über ihre politische Zugehörigkeit und Ideologie. Die Erziehung und Prägung in der Kindheit, üben Einfluss auf die Gewichtung sozialer TeilIdentitäten aus. Später spielt das Beziehungsgeflecht, das eine Person aufbaut, eine wichtige Rolle, da gruppenspezifische Werte und Normen übernommen werden, die wiederum die ethnisch, moralische Einstellung prägen. ${ }^{9}$ Jede soziale Identität eines Menschen reflektiert die gesellschaftlichen Strukturen. Dies gilt auch für einzelne Teil-Identitäten wie z.B. der familiären, beruflichen, religiösen, ethnischen, nationalen und geschlechtlichen Identität. ${ }^{10} \mathrm{Da}$ jedes Individuum individuell wahrnimmt und sich entsprechend ausdrückt, entsteht ein vielseitiges Bild der gesellschaftlichen Organisation. Die Schwierigkeit für Flüchtlinge besteht darin, während des Akkulturation-Prozesses, die Normen, Werte und Symbole der Gastkultur/des Aufnahmelandes zu erkennen und zu verstehen, um sie in ein Verhältnis zu der Heimatkultur setzen zu können. Die anfängliche Ausgrenzung von Flüchtlingen und Asylanten z.B. in Aufnahmezentren, erschwert diesen Vorgang noch, da zu wenig

8 ebd S. 77

${ }^{9}$ ebd S. 78ff

${ }^{10}$ ebd. S.297 
Interaktikonen mit der Aufnahmegesellschaft stattfinden. ${ }^{11}$ Der fiktive unbegleitete minderjährige Flüchtlingsjunge Djadi wird von dem Sozialarbeiter Jan nach einem Besuch in der Jugendhilfe spontan mit nach Hause genommen. Wie lange er sich zuvor schon in Deutschland aufgehalten hat, erfährt man in dem Roman nicht. Sein Verhalten und seine Unsicherheit in der Wohngemeinschaft zeigen aber, dass ihm die Aufnahme-Gesellschaft noch sehr fremd ist.

Literatur zum Thema «Identität» baut meist auf der epigenetischen Theorie der Persönlichkeitsentwicklung von Erik H. Erikson auf. Laut Erikson durchläuft jede Person im Laufe ihres Lebens verschiedene Stufen, die gekoppelt an Krisen oder Konflikte sind. Die acht Stufen laufen linear ab und können nicht übersprungen werden. ${ }^{12}$ Für diese Arbeit sind vor allem die vierte und fünfte Phase von Relevanz, da sie dem Alter der fiktiven Romanfigur Djadi entsprechen. Die vierte Stufe umfasst die Zeit eines Schulkindes von sechs bis zwölf Jahren. Es ist die Latenzphase «Industry vs Inferiority» oder «Werksinn versus Minderwertigkeitsgefühl». Das Kind erkennt seine eigenen Interessen und realisiert, dass es sich von anderen unterscheidet. Die Herausforderung dieser Stufe ist die Entwicklung der Kapazität für Fleiß. Grundsätzlich wollen Kinder dieses Alters zeigen, welche Fähigkeiten in ihnen stecken und streben nach Selbständigkeit. Erfahren Kinder Anerkennung und Bestätigung, sind sie motiviert und eifrig. Werden sie hingegen oft gerügt, eingeschränkt oder nicht beachtet empfinden sie sich als unnützlich, dumm und inkompetent. Während dieser Stufe ist der Einfluss der KollegInnen, der Nachbarn und Lehrer am höchsten. Wie in jeder Phase gilt es eine Balance zwischen den positiven und negativen

\footnotetext{
${ }^{11}$ Florian Lampersberger, 2016, Auswirkung von Flucht auf Identität, https://www.researchgate.net/publication/304495036, S. 11

${ }^{12}$ C. George Boeree, 2006, Personality Theories, Übersetzung D. Wieser, 2006, https://kritisches-netzwerk.de/sites/default/files/george boeree -

_persoenlichkeitstheorien_nach_erik_h_erikson_- personality theories__ 20 seiten.pdf, S. 6
} 
Erlebnissen und Gefühlen zu finden. Kinder müssen auch lernen, dass sie Dinge erledigen oder einüben müssen, deren Sinn und Zweck sich ihnen entzieht. Zudem werden sie mit den Anforderungen sozialer Fähigkeiten konfrontiert, die die Gesellschaft an sie stellt. Junge Menschen, die in dieser Phase flüchten müssen, erleben sehr oft Rassismus, Sexismus und andere Formen der Diskriminierung. Das sind Quellen für Minderwertigkeitsgefühle, die es erschweren, eine gesunde Balance zwischen Motivation, Eifer und Unterlegenheit zu finden. Das Selbstwertgefühl und die Ich-Identität leiden stark darunter.

Die fünfte Stufe beginnt mit der Pubertät und endet zwischen achtzehn und zwanzig Jahren. E. Erikson nannte sie «Identity vs Role Confusion» oder «Ich-Identität versus Rollenverwirrung». In dieser Zeit realisiert der Jugendliche, dass er verschiedene soziale Rollen ausübt. Er kann z.B. FreundIn, StudentIn und Schwester oder Bruder sein. Viele erleben in diesem Zeitraum eine Identitätskrise. Die Fragen «Wer bin ich?» und «Wie passe ich in die mich umgebende Gesellschaft?» stehen im Zentrum dieses Prozesses. Diese Lebensphase ist geprägt durch die Dynamik eines biologischen, psychologischen und soziologischen Übergangs, die mit inneren und äußeren Trennungserfahrungen verbunden ist. Eine zu starke einseitige Entwicklung der Ich-Identität führt dazu, dass eine Person sich in einer Rolle extrem verfestigt, zum Fanatiker wird und kaum noch Platz für Toleranz übrigbleibt. Überwiegt die Rollenverwirrung und mangelt es Jugendlichen an dem Selbst - der Ich-Identität - oder wird das Bedürfnis danach einfach negiert, besteht für sie die Gefahr, in ein Suchtverhalten abzugleiten. Eine andere Art von «Flucht» stellt sich dar, indem sich der junge Mensch einer Gruppe anschliesst, die eine detaillierte Identität bereitstellt. Für diese Gruppen gelten ganz strikte Regeln, die oft an eine hohe Ideologie geknüpft sind, wie z.B. bei Sekten oder militanten Gruppierungen. Das Individuum wird einfach zu einem Glied einer Masse. Kann diese wichtige fünfte Phase hingegen erfolgreich ausbalanciert werden, erreicht man laut E. Erikson, trotz 
seiner Unvollkommenheit, eine gewisse Glaubwürdigkeit (fidelity). Die Voraussetzungen, dass das Individuum einen Platz in der Gesellschaft findet und seinen Beitrag leisten kann, sind damit geschaffen. ${ }^{13}$ Die Ich-Identität verlangt eine Persönlichkeitsreife. Sie besteht aus der Fülle der gemachten Kindheitserfahrungen und bereitet auf die Aufgaben des Erwachsenenlebens vor. Für junge Flüchtlinge, kommt erschwerend hinzu, dass die verschiedenen Teile der sozialen und personalen Identität nicht mehr in ein kohärentes und kontinuierliches Selbst-Konzept integriert werden können. ${ }^{14}$

\section{Analyse des Buches „Djadi, Flüchtlingsjunge“ von Peter Härtling}

Härtling erzählt in seinem Buch die Geschichte eines 11jährigen Jungen namens Djadi, der wegen des Krieges in seinem Heimatland Syrien, mit seiner Familie, nach Deutschland flieht. Auf der Flucht über das Mittelmeer muss er miterleben, wie seine Eltern ertrinken. (S.31) Seine drei Schwestern sind zu diesem Zeitpunkt vermutlich schon tot. Er erreicht Frankfurt gemeinsam mit anderen syrischen Flüchtlingen. In der Jugendhilfe trifft er auf Jan, einen deutschen Sozialarbeiter, der ihn spontan mit nach Hause nimmt. Jan wohnt mit seiner Frau Dorothea, einer Psychologin, und zwei anderen Ehepaaren in einer Wohngemeinschaft. Die Mitglieder der WG sind zuerst überrascht über den neuen Gast, dann aber bereit, den Jungen bei sich aufzunehmen. (S.5-8) Härtling entscheidet sich beim Zufluchtsort für Djadi bewusst, für eine Wohngemeinschaft. Er will damit zum Ausdruck bringen, dass ein Flüchtling immer in eine ganze Gemeinschaft hineinwachsen muss und dass jeder Mensch des Aufnahmelandes, der bei sich ist,

\footnotetext{
${ }^{13}$ Ebd S.11-14

${ }^{14}$ Florian Lampersberger, 2016, Auswirkung von Flucht auf Identität, https://www.researchgate.net/publication/304495036, S. 14
} 
die Kraft und Wärme hat, ein Flüchtlingskind zu begleiten. ${ }^{15}$ Die drei Paare der Wohngemeinschaft unterstützen Djadi auf seinem langen Weg der Eingliederung in die deutsche Gesellschaft. Die Erinnerung an seine Vergangenheit, die schrecklichen Kriegs- und Fluchterlebnisse, belasten Djadi sehr und erschweren es ihm, sich auf die neue Situation einzulassen. (z.B. S.18,29,44,64,79) Zu dem pensionierten Lehrer der WG mit dem Spitznamen Wladi, baut Djadi besonders schnell eine gute Beziehung auf. Zu der Rolle dieser Romanfigur sagte Härtling in einem Interview folgendes:

«Es braucht Verständnis für kleine Menschen, die Dinge erleben, die große Menschen anstellen. Kinder bewältigen das nicht, diese Erlebnisse stürzen in sie rein, sie schleppen all das mit. Den Wladi habe ich für diese WG erfunden, weil ich jemanden brauchte, der Djadi auffängt. Einen, der ein bisschen reagiert wie ich. Denn aus geteilter Angst entsteht Nähe». ${ }^{16}$

Mit Waldis Verständnis gelingt es Djadi, sich langsam in der neuen Welt zurechtzufinden. Sein Urvertrauen wurde durch die Geschehnisse der vergangenen Jahre stark erschüttert. Seine IchIdentität litt darunter und muss wiederaufgebaut werden. Ein Kind entwickelt seine Identität, seine körperliche und psychische Ganzheit, solange es nicht traumatisiert wird. Die Summe aller bewusst und unbewusst gemachten Lebenserfahrungen helfen ihm dabei seine Identität zu entwickeln. ${ }^{17}$ Djadis verlorene Ich-Identität drückt Härtling in diesem Roman z.B. mit dem mangelnden Selbstvertrauen des Jungen aus. Er beschreibt, wie der Elfjährige sich unsicher verhält, wenn er mit unbekannten oder

\footnotetext{
${ }^{15}$ Kristina Kroll, 2017, «Djadi, Flüchtlingsjunge» im Unterricht, Beltz \& Gelberg Verlag, Weinheim, S. 15

16 Anne Haeming, 2016, Kinderbuchautor Peter Härtling "Aus geteilter Angst entsteht Nähe", https://www.spiegel.de/kultur/literatur/autor-peter-haertling-kinderbuch-djadifluechtlingsjunge-a-1112268.html

${ }^{17}$ Franz Ruppert, 2016, Wer bin ich, who am I, 3. Internationaler Kongress, PDF, https://franz-ruppert.de/de/downloads/send/17-gehaltene-vortrge-deutsch/267identitaet-und-trauma
} 
unangenehmen Situationen konfrontiert wird. Er verkriecht sich unter dem Sofa oder versteckt sein Gesicht hinter den Händen. (z.B. S.9,12,13,27,39,69) Der Leser erfährt, dass Djadi viel Zeit braucht, sich wieder zu spüren und Vertrauen zu seinen Mitmenschen zu fassen. Die Ich-Identitäts-Entwicklung ist eng verknüpft mit dem „Wir-Sein“, da kein Mensch alleine überlebensfähig ist. ${ }^{18}$

Die Bewohner der WG sind herausgefordert, einen Zugang zu Djadis verwundeter Seele zu finden. Schritt für Schritt lernt der syrische Junge die neuen Werte und Normen der deutschen Gesellschaft kennen. Durch die Flucht müssen Flüchtende alles zurückgelassen, was ihnen vertraut war; die vertraute Umgebung, die vertrauten Menschen und die vertraute Kultur. Die Heimat vermittelt ein Gefühl von Zugehörigkeit, Identifikation, und bildet die soziale und kulturelle Identität. ${ }^{19}$ Djadi bleibt nach der Flucht nur seine personale Identität übrig.

Das Sozialamt, die Ausländerbehörde, das Jugendamt, der Amtsarzt und die Schule sind verschiedene Stationen in diesem Roman, mit denen Djadi konfrontiert wird. Schon am Anfang der Geschichte wird geschildert, wie die deutsche Bürokratie Jans Bemühungen erschwert, die Pflegeelternschaft für Djadi zu übernehmen. Die ganze WG muss zum Beispiel bei den Behörden ein polizeiliches Führungszeugnis vorlegen. (S.11,37) Die Kommunikation mit Djadi ist erschwert, da er zu Beginn kein Wort Deutsch spricht. Auch später, als er die Sprache besser beherrscht, bleibt er manchmal sprachlos. (z.B. S.43,63) Das irritiert die Mitglieder der Wohngemeinschaft, so dass sie öfters nicht wissen, wie sie am besten mit dem Jungen umgehen sollen. Für diese Situationen voller Selbstzweifel hat Härtling in diesem Roman die fiktive Figur Wladi geschaffen. Der pensionierte Lehrer schenkt allen immer wieder Zuversicht. (S.19, 67, 77) Gegen Ende des

\footnotetext{
18 ebd

${ }^{19}$ Verena Vordermayer, 2012, Identitätsfalle oder Weltbürgertum, Verlag für Sozialwissenschaften, D-Wiesbaden, S. 49
} 
Buches erfährt der Leser, dass Wladi selbst als Kind flüchten musste und deshalb so viel Verständnis für Djadis Situation aufbringen kann. (S.77) Djadis Suche nach seiner Ich-Identität kommt in verschiedenen Sequenzen des Romans zum Ausdruck. Als erstes malt er ein Bild seiner Familie. Einen großen, dünnen Mann, daneben eine kleinere Person, die eine Frau sein könnte. Sie trägt ein Kleid und ein Kopftuch. Neben dem Paar stehen drei kleine Menschen und mit einem Abstand, ganz alleine, eine vierte sehr kleine Person. Als Kordula auf diese zuletzt gezeichnete Figur zeigt und fragt: „Djadi?““ antwortet er leise mit: „Ich!“. (S.24) Dabei kommen ihm fast die Tränen. In der Nacht hört Detlef Djadi stöhnen, reden und weinen. Am nächsten Tag fragt er ihn, wie es ihm gehe. Zur Antwort bekommt er: „Gut auch schlecht.“ Damit bringt Härtling die innere Zerrissenheit Djadis zum Ausdruck. Die Vertrautheit Syriens ist ihm genommen worden und in Deutschland ist er noch nicht richtig angekommen. An anderer Stelle sagt Djadi: „Ich bin falsch. [...] Ich passe nicht.“ (S.63,81) Er fühlt sich nicht dazugehörig, sondern fremd. Im Kapitel „Erschrocken“ beschreibt Härtling, dass Djadi in der Stadt zwei der Schlepper zu erkennen glaubt. Dies versetzt ihn in Panik. (S.30) Er rennt nach Hause und hat und kann kaum noch atmen. Da Djadi noch nicht auf Deutsch erklären kann, was ihn so erschreckt hat, bietet ihm Wladi ein Blatt Papier und einen Stift an. Djadi malt erzählend eine schlimme Episode, die sich auf dem Fluchtweg über das Mittelmeer ereignete. In einem Boot mit viel zu vielen kleinen Menschen stehen zwei große Männer. Einer drückt mit seiner großen Hand im Meer schwimmende Köpfe unter Wasser. „Weg“, sagt Djadi, „weg!“ Sein kleiner, schmächtiger Körper bebt und sein Atem stockt. Auch Wladi hat Tränen in den Augen. Er fragt: „Deine Mama? Dein Papa?“ Djadi verbirgt sein Gesicht in den Händen. (S.31) Dieses traumatische Erlebnis, das Härtling hier beschreibt, erklärt, weshalb Djadi seine Ich-Identität verloren hat.

Als Djadi mit seinen Pflegeeltern Jan und Dorothea mit der Bahn in die Ferien fährt, bezeichnet die Schaffnerin Jan in einem 
Gespräch als Djadis Papa. Djadi reagiert verwirrt. Dorothea fragte ihn, ob dies für ihn ein Problem sei. Nach langem Nachdenken verneint er die Frage und meint: „Ich habe einen Papa und einen Papa jetzt hier." (S.50) Kinder kommen aus verschiedensten Gründen in die Obhut von Pflegeeltern. Dieser Neubeginn verunsichert ihr Ich-Gefühl. Sie haben Angst sich selbst zu verlieren. ${ }^{20}$ Später in der Pension auf der Insel Juist, bleibt Djadi vor dem Spiegel im Gästezimmer stehen und betrachtet sein Spiegelbild. „Zwischen ihm und dem Jungen im Spiegel gibt es eigentlich nur eine Verbindung: Die Angst und eine plötzliche Stille." (S.57) Mit dieser Szene verdeutlicht Härtling ein weiters Mal, dass Djadi sich erst wiederfinden muss und sich selbst bisweilen fremd ist. Unsicherheit und Angst sind Djadis ständigen Begleiter. Als Wladi und seine Frau Kordula zu Besuch auf die Insel kommen, verbringen sie den ersten Abend gemeinsam in einem Restaurant. Am Nachbartisch ereifern sich Gäste laut über die Asylanten Deutschlands. Djadi hört angestrengt zu und fragt, ob er sich als Asylant zu erkennen geben soll. Wladi unterstützt seine Idee sofort. So geht Djadi zum Tisch nebenan und verkündet, dass er auch ein Asylant sei. (S.76) Für diese Aktion braucht es schon eine ganze Portion Mut und zeigt, dass das Selbstwertgefühl Djadis langsam wächst. Härtling nimmt hier die Themen Vorurteile und Rassismus auf. Nach den Ferien tritt Djadi in die Schule ein. Schon am ersten Tag muss er sich gegen seinen Banknachbarn durchsetzen, der ihn in der Pause als falschen Hund und Scheißaraber bezeichnet. Auf diese Provokation reagiert Djadi, indem er auf David einschlägt. Die zwei Jungs prügeln sich, bis Lehrer Köhler dazwischen geht und die Situation beruhigt. $($ S.94,95) Am nächsten Tag schwänzt Djadi die Schule. Er will sich dieser Herausforderung nicht mehr stellen. Er spürt seine Defizite gegenüber den anderen Schülern und zweifelt an seinen Fähigkeiten. Er fühlt sich einmal mehr fremd und fehl am Platz.

\footnotetext{
${ }^{20}$ Irmela Wiemann, 2011, »Was kann bei so einem Papa schon aus mir werden?«, IdentitätPflegekinder-Wiemann.pdf
} 
Immer wieder zeigt Härtling, wie wenig es braucht, um das vorsichtig aufgebaute Ich-Gefühl wieder zu zerstören oder ins Wanken $\mathrm{zu}$ bringen. Schließlich schafft es Djadi mit der Unterstützung aller WG-Mitglieder in der Schule Fuß zu fassen und entwickelt sich zu einem guten Schüler. (S.100,101) Nachdem Djadi schon vier Jahre in Deutschland ist, stirbt sein bester Freund Wladi. Bei der Beerdigung tritt ein Mann an Djadi heran und sagt ihm: „Du bist also Wladis Freund, der ihm seine letzten Jahre leicht machte.“ (S.115) Diese Worte freuen Djadi sehr und stärken sein Selbstwertgefühl. Er erlebt, wie die WG-Mitglieder Kordula und ihm in dieser schweren Zeit besonders viel Aufmerksamkeit schenken. Dieses Zusammenstehen in schweren Momenten stärkt auch Djadis soziale Identität wieder. Das Wir-Gefühl wird wieder gestärkt in ihm. Härtling beschreibt nun sehr schön, wie er dadurch langsam auch seine Trauer über den tragischen Verlust seiner Eltern und Geschwister zulassen kann, indem er zwei Steine mit einem P (Papa) und einem M (Mama) bemalt und sie auf mit auf dem Grab von Wladi platziert. (S.115,116)

\section{Schlusswort}

Peter Härtling beschreibt in diesem Roman auf eindrückliche Art und Weise, wie viele Herausforderungen an geflüchtete Kinder gestellt werden, die alles verlieren, was ihnen lieb und teuer war. Auch das Zurechtfinden in einer neuen Kultur verlangt viel Energie. Zudem stellt die Aufnahmegesellschaft von Beginn weg Anforderungen an die Geflüchteten. In dem Roman «Djadi, Flüchtlingsjunge» zeigt Peter Härtling, dass die Neuankömmlinge primär einfach einmal Zuwendung und Zeit brauchen, um wieder $\mathrm{zu}$ sich $\mathrm{zu}$ finden. Erst dann sind sie fähig ihr Potenzial zu entwickeln. Die Aussage von Peter Härtling, die bereits im ersten Abschnitt dieser Arbeit erwähnt wurde widerspiegelt sich in seinem Roman: 
„Es hätte mir als Kind geholfen, wenn man mich nicht als ein Niemand, der integriert werden muss, behandelt hätte -sondern als Mensch.» ${ }^{21}$

Mit der Figur Wladi demonstriert er in dieser Geschichte, was er damit meint.

\section{Literaturverzeichnis:}

- Abels Heinz, 2006, Identität, VS Verlag für Sozialwissenschaften, Wiesbaden.

- Kroll Kristina, 2017, «Djadi, Flüchtlingsjunge» im Unterricht, Beltz \& Gelberg Verlag, Weinheim.

- $\quad$ Müller Bernadette, 2011, Empirische Identitätsforschung, VS Verlag für Sozialwissenschaften, Wiesbaden.

- Wiemann Irmela, 2011, »Was kann bei so einem Papa schon aus mir werden?«, Identität-Pflegekinder-Wiemann.pdf

- Vordermayer Verena, 2012, Identitätsfalle oder Weltbürgertum, Verlag für Sozialwissenschaften, D-Wiesbaden.

- Z Zirfas Jörg, 2010, Schlüsselwerke der Identitätsforschung, VS Verlag für Sozialwissenschaften, Wiesbaden.

\section{Internetseiten:}

- $\quad$ https://www.forschung-frankfurt.uni-

frankfurt.de/36050401/forschung-frankfurt-ausgabe-2-2005-mitleid-fur-daseigene-kind-in-mir.pdf. http://www.haertling.de/

\section{Djadi.pdf}

\section{https://www.beltz.de/fileadmin/user_upload/PI_Haertling-}

${ }^{21}$ Franziska Annabelle Lindner, 2016,

https://www.beltz.de/fileadmin/user_upload/PI_Haertling-Djadi.pdf 
- $\quad$ https://www.researchgate.net/publication/304495036.

- $\quad$ https://kritischesnetzwerk.de/sites/default/files/george_boeree__persoenlichkeitstheorien_nach_erik_h_erikson_-_personality_theories__20_seiten.pdf.

- $\quad$ https://www.researchgate.net/publication/304495036.

- $\quad$ https://www.spiegel.de/kultur/literatur/autor-peter-haertlingkinderbuch-djadi-fluechtlingsjunge-a-1112268.html

- $\quad$ https://franz-ruppert.de/de/downloads/send/17-gehaltenevortrge-deutsch/267-identitaet-und-trauma 